\title{
Laser-assisted Surgical Excision of Fibroepithelial Polyp with Periosteal Lasing: A Case Report
}

\author{
Nitesh Tewari ${ }^{1}$, Morankar Rahul ${ }^{2}$, Deepika Mishra ${ }^{3}$
}

\begin{abstract}
Introduction: Reactive lesions of gingiva have been shown to exhibit varied features even with similar etiologic irritants. A fibroepithelial polyp is characterized by pink, red, or white knob-like growth with sessile or pedunculated base with a prevalence of $1-2 \%$ and a higher female predilection.

Case description: A 13-year-old female patient reported with chief complaint of an enlarging, painless soft mass in lower front region of the jaw since last the 6 months. Intraoral examination revealed firm, nontender, pinkish-white sessile lesion of $1.5 \times 1.5 \times 0.5 \mathrm{~cm}$ on interdental papilla and attached gingiva on both labial and lingual sides in relation to tooth 32 and 33 . A $940 \mathrm{~nm}$ diode laser-assisted surgical excision and lasing of periosteum were performed under local anesthesia. There was no recurrence after 18 months with complete restoration of normal form and esthetics. Histopathology was suggestive of fibroepithelial polyp.

Conclusion: The clinicians need to be aware of the clinical, histopathological features, and management of reactive mucosal lesions. Diode laser is an important aid in performing surgical excision of these lesions with minimal patient discomfort, increased operator control, and good postoperative results.

Keywords: Fibroepithelial polyp, Fibroma, Laser excisional biopsy.

Journal of South Asian Association of Pediatric Dentistry (2020): 10.5005/jp-journals-10077-3048
\end{abstract}

\section{INTRODUCTION}

Oral epithelium acts as the first line of defense to various infections with an active role in innate host response. ${ }^{1}$ One of its most significant properties is its regenerative ability and rapid repair. However, this equilibrium shifts and leads to reactive overgrowths of oral mucosa. ${ }^{1,2}$ The reactive lesions of gingiva have been shown to exhibit varied features even with similar etiologic irritants. ${ }^{3}$

Daley et al. suggested the term "focal fibrous hyperplasia" (FFH), as it describes the pathogenesis and clinical appearance of lesion. ${ }^{4}$ It was also found to be preferable over the term "fibroma" or irritation fibroma. ${ }^{1,4}$ Another terminology of similar nature is "localized hyperplastic reactive lesions" of gingiva which represents a group of lesions with overlapping clinical and histologic features. ${ }^{5}$ Localized hyperplastic reactive lesions of gingiva can be further classified as (1) pyogenic granuloma, (2) peripheral ossifying fibroma, (3) peripheral giant cell granuloma, and (4) $\mathrm{FFH}^{5}$

A fibroepithelial polyp is characterized by pink, red, or white knob-like growth with sessile or pedunculated base. ${ }^{1}$ They can arise anywhere on the mucosa of the oral cavity and act as a concern for patient's aesthetics and smile. ${ }^{6}$ It has a prevalence of 1 to $2 \%$ and a higher female predilection (2.2:1). ${ }^{1,2,6}$

Surgical excision is the recommended treatment due to benign nature of the lesion; however, a long-term follow-up is recommended to monitor the recurrence. Electrocautery, lasers (Nd: YAG laser, diode laser, and flash lamp-pulsed dye laser), cryosurgery, and intralesional injections of sclerosing agents and/or corticosteroids have been attempted in similar lesions with variable success. ${ }^{6-8}$ This article comprised of a case of fibroepithelial polyp treated by diode laser-assisted excision with 18 months follow-up.

\section{Case Description}

A 13-year-old female patient reported with chief complaint of an enlarging, painless, soft mass in lower front region of jaw. Lesion
1,2Department of Pedodontics and Preventive Dentistry, Centre for Dental Education and Research, All India Institute of Medical Sciences, New Delhi, India

${ }^{3}$ Department of Oral Pathology, Centre for Dental Education and Research, All India Institute of Medical Sciences, New Delhi, India

Corresponding Author: Nitesh Tewari, Department of Pedodontics and Preventive Dentistry, Centre for Dental Education and Research, All India Institute of Medical Sciences, New Delhi, India, Phone: +91 9839311058, e-mail:dr.nitesht@gmail.com

How to cite this article: Tewari N, Rahul M, Mishra D. Laser-assisted Surgical Excision of Fibroepithelial Polyp with Periosteal Lasing: A Case Report. J South Asian Assoc Pediatr Dent 2020;3(2):88-91.

Source of support: Nil

Conflict of interest: None

was first noticed on gums 6 months ago, and it slightly increased in size. It has been a major concern due to poor aesthetics. Medical history was noncontributory, and there was no history of trauma to the region. Intraoral examination revealed firm pinkish white sessile lesion of $1.5 \times 1.5 \times 0.5 \mathrm{~cm}$ on interdental papilla and attached gingiva on both labial and lingual sides in relation to tooth 32 and 33 (FDI notation). Labial and lingual growths seemed to join in the interdental space between two teeth (Figs $1 \mathrm{~A}$ and $\mathrm{B}$ ). There was no tenderness on palpation in soft tissue mass, and no mobility was observed in 32 and 33 . The child had permanent dentition with good oral hygiene and no dental caries. Intraoral periapical radiograph show normal 32 and 33 with no resorption of alveolar bone crest (Fig. 1C). Clinical diagnosis of fibroma was made. Parental consent was obtained before commencement of treatment, and excisional biopsy was planned under local anesthesia.

Diode laser (Biolase, USA-940 nm, power settings of 2 Watt, continuous contact mode and the manufactured supplied standard 

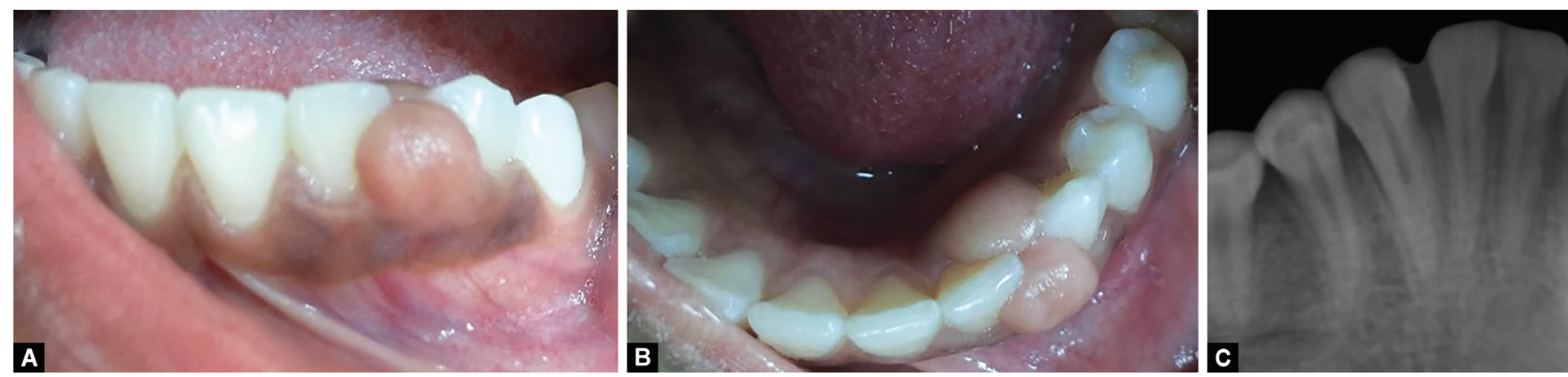

Figs $1 \mathrm{~A}$ to $\mathrm{C}$ : (A) Pinkish-white sessile lesion of $1.5 \times 1.5 \times 0.5 \mathrm{~cm}$ on interdental papilla and attached gingiva on both labial and lingual sides in relation to 32 and 33; (B) Occlusal view showing labial and lingual growths joining in the interdental space between the two teeth; (C) Intraoral periapical radiograph showed normal 32 and 33 with no resorption of alveolar bone crest
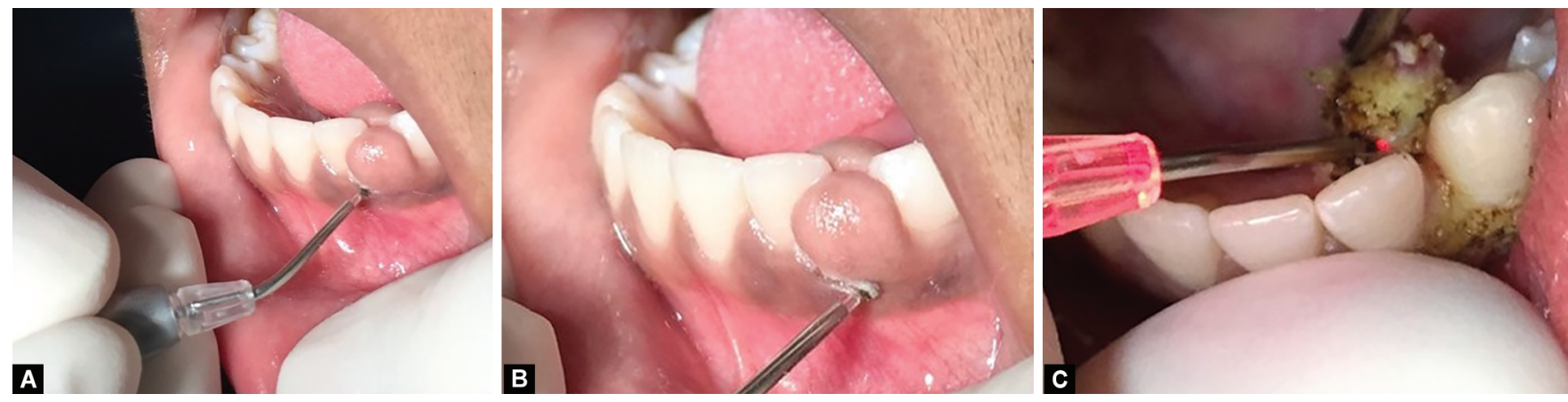

Figs $2 \mathrm{~A}$ to $\mathrm{C}$ : (A and B) Diode laser-assisted surgical excision under local anesthesia; $(C)$ The lasing of periosteum for removal of tissue tags

initiated tip of 300 microns diameter) - assisted surgical excision under local anesthesia was done. Periosteum under the excised tissue was "lased" using the same laser tip in a contact mode, using $1.5 \mathrm{~W}$ power and moving rhythmically to cover the entire surface of the exposed periosteum. Tissue was sent for histopathological examination per the protocol. The procedure was completely blood less with complete removal of tissue tags from underlying periosteum (Fig. 2). The patient was discharged after counseling regarding maintenance of oral hygiene. Healing was uneventful as seen in follow-ups immediately postoperatively (Figs 3A and B) and at 7 days (Figs $3 C$ and D).

The lesion did not show any recurrence after 18 months, with complete restoration of normal form and aesthetics (Fig. 4).

Low-power view (10x) of the section showed hyperplastic connective tissue stroma covered by mildly hyperplastic epithelium showing spiky rete processes (Figs $5 \mathrm{~A}$ and B). High-power view showed stratified squamous type of epithelium showing acanthosis in few areas overlying the irregularly interlacing dense bundles of collagen fibers with spindle- to stellate-shaped fibroblasts and interspersed endothelial-lined blood vessels with and without red blood cells (Fig. 5C). The features were suggestive of fibroepithelial polyp.

\section{Discussion}

Reactive fibroepithelial lesions of oral mucosa are relatively rare with a prevalence of $1-2 \%{ }^{1,2,5}$ They are more common in females in third to fourth decade of life. A clear etiopathogenesis in most cases has resulted in multiple nomenclatures, such as irritation fibroma and denture epulis. ${ }^{1,3}$ However, the literature has reported cases of fibroepithelial lesions with obscure etiology as well. ${ }^{1,2}$ In the present case, the lesion has occurred in the absence of any irritant or stimuli (sharp tooth/teeth, faulty restoration, chemical irritant, biting habit, history of traumatic extraction or poor oral hygiene). However, patient's age and associated hormonal changes may be attributed to development of such lesion. Buchner has reported eruption of teeth and related irritation of mucoperiosteum as possible cause of reactive lesions of gingiva. ${ }^{5}$ Excisional biopsy as first line of treatment in reactive fibroepithelial lesions of gingival has been advocated along with the histopathological examination of the lesion. ${ }^{4,5}$

In the recent years, the safety and efficacy of diode lasers in soft tissue surgical procedures has been well established., ${ }^{7,8}$ The benefits of treating a lesion with diode laser include negligible postoperative inflammation, damage, enhanced healing, and reduced postoperative pain. ${ }^{9}$ American Academy of Pediatric Dentistry has also given guidelines stating the advantages of performing laser-assisted procedures in children and adolescents following standard safety protocols. ${ }^{10}$ Use of lasers in surgical excision precludes the preoperative anxiety associated with surgical procedures in children. ${ }^{10,11}$ It further reduces the need for nerve block anesthesia, with most soft tissue surgeries requiring only topical anesthesia. Its safety also makes it helpful in soft tissue surgeries in neonates and infants. ${ }^{11}$

The $940 \mathrm{~nm}$ diode lasers used in this case is advantageous due to low thermal effect on depth of lased tissue. This property is helpful in preserving histopathological samples for better evaluation., 70 Additionally, it provides relatively bloodless intraoperative phase and uneventful healing period with minimal swelling and scarring. ${ }^{10,11}$ Similar advantages were noticed in present case as well. 

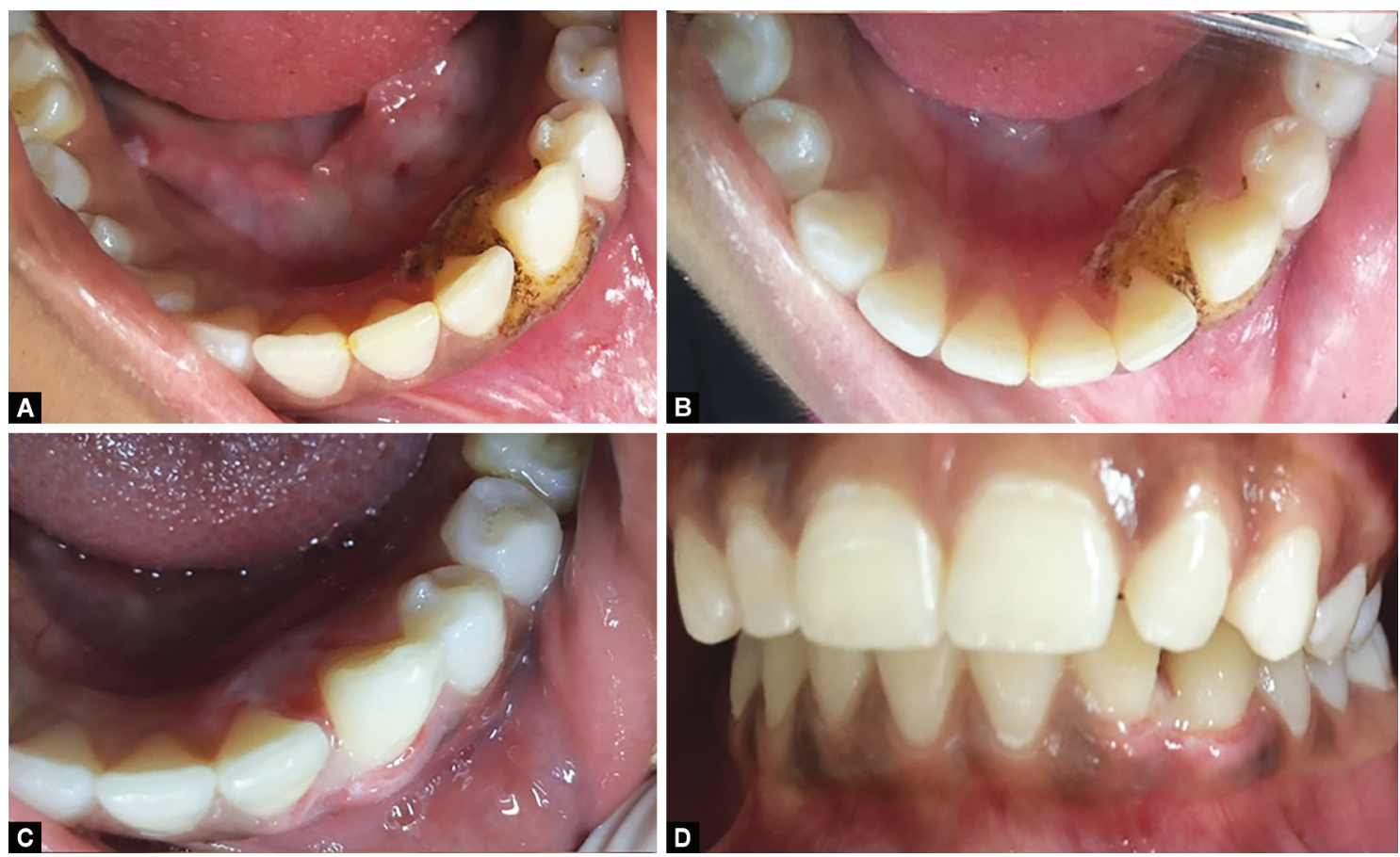

Figs 3A to D: (A and B) Immediate postoperative results; (C and D) One week postoperatively

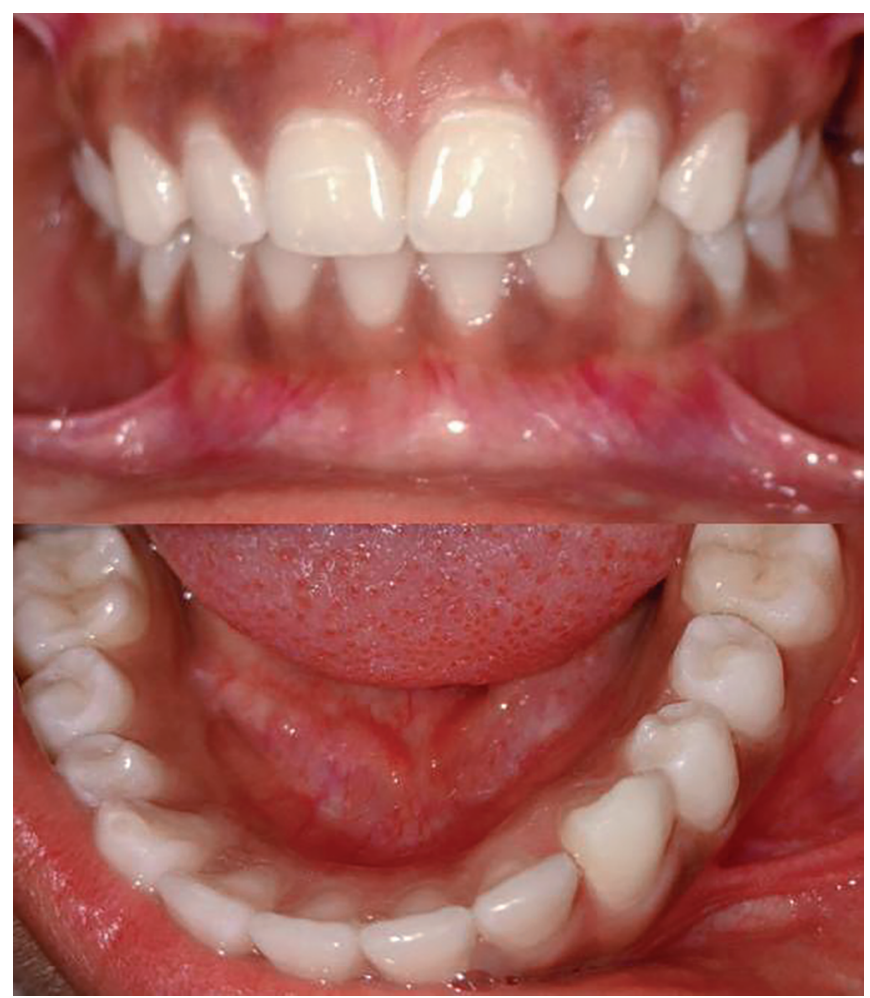

Fig. 4: Eighteen months follow-up

Laser creates locally sterile conditions, and hence no prophylactic antimicrobial agent is required in most cases. ${ }^{11}$

Histopathological features of fibroepithelial polyp comprise of stratified squamous epithelium covering hyperplastic connective tissue stroma with collagen bundles and blood vessels. ${ }^{5,6}$ Histopathology of present case was also found to be like description of other researchers. The histopathology forms the only confirmatory method for establishing the diagnosis for reactive hyperplastic lesions. Although clinically this lesion looked like an induced hyperplastic gingival lesion (puberty, drug induced, etc.), the lack of drug history and presence of bleeding ruled these out. Even its probable hormonal etiology is related to localized hyperplasia unlike the usual puberty gingivitis. Other entities, such as fibroma, epulis, pyogenic granuloma, and ossifying fibroma, were also ruled out based on the continuum of clinical and histological features.

The surgical technique followed in present case was novel and based on two important principles: (1) complete excision of the fibrous lesion with preservation of tissue for histopathology and (2) a second lasing of periosteum at 1.5 watt for eradication of any attached tissue tag. Since the reactive lesions are associated with high recurrence, this step is being advocated to minimize the chances of regrowth. This protocol was fairly successful in present case with no recurrence in a follow-up period of over 18 months.

\section{Conclusion}

The clinician needs to be aware of reactive lesions of gingiva, especially fibroepithelial polyp along with their histopathological features and management. Diode laser is an important aid in performing surgical excision of these lesions with minimal patient discomfort, increased operator control, and good postoperative esthetic results. Protocol involving tissue preservation and lasing of periosteum is an innovative method for excision of reactive lesions and can be explored through future research. 


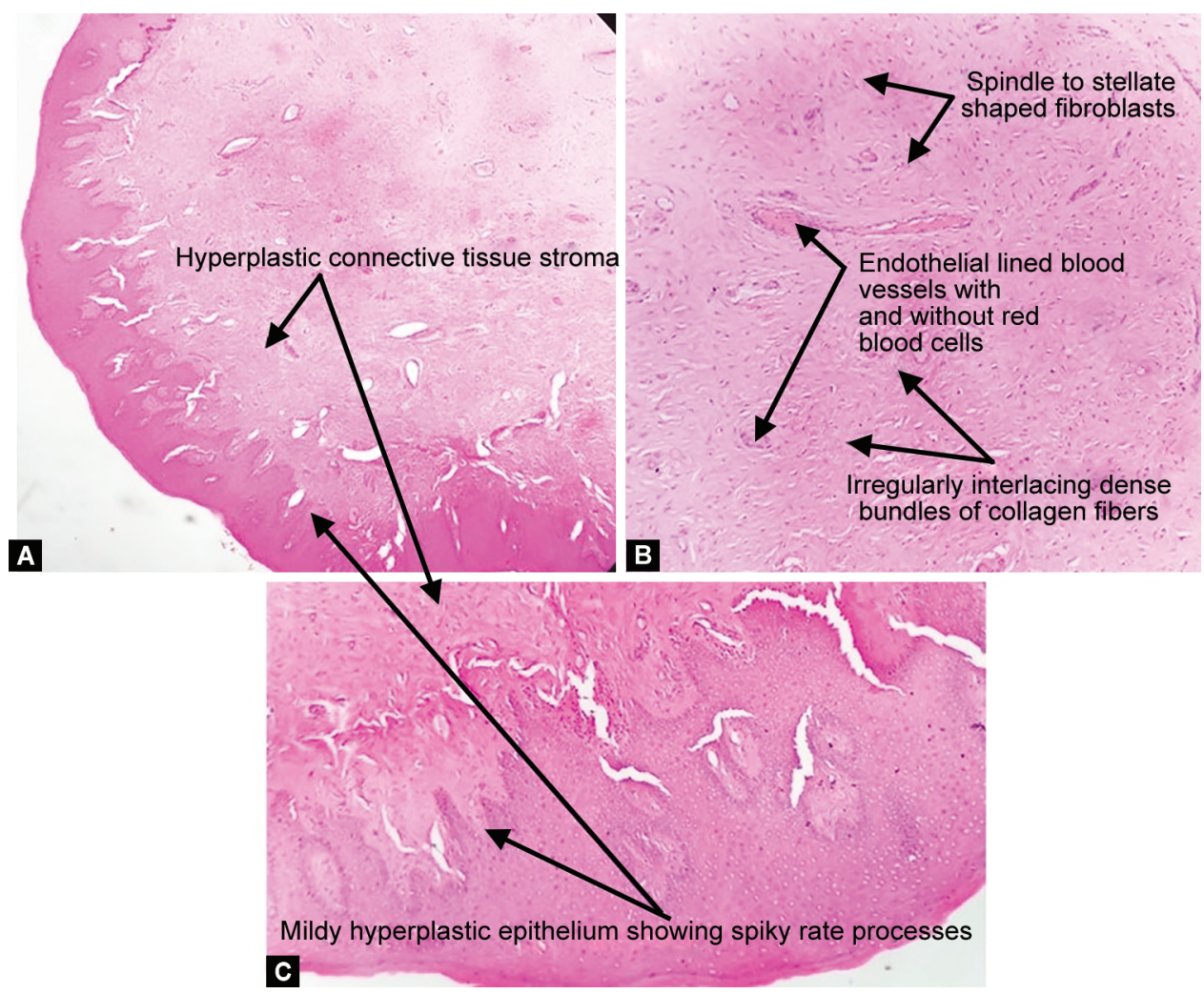

Figs 5A to C: (A and B) The low-power view $(\times 10)$ of the section; $(C)$ High-power view $(100 \times)$ of the section

\section{REFERENCES}

1. Neville BW, Damm DD, et al. Soft tissue tumors. In: Oral and Maxillofacial Pathology. 3rd ed., Philadelphia, PA, USA: Saunders; 2002. pp. 507-512.

2. Shafer Hine, et al. Benign and malignant tumors of oral cavity. In: Rajendran R, Shivapathasundaram B. Shafer's Textbook of Oral Pathology. New Delhi, India: Elsevier; 2007. pp. 178-180.

3. Eversole LR, Rovin S. Reactive lesions of the gingiva. J Oral Pathol 1972;1(1):30-38. DOI: 10.1111/j.1600-0714.1972.tb02120.x.

4. Daley T, Wysocki G, Wysocki PD, et al. The major epulides: clinicopathological correlations. J Can Dent Assoc 1990;56(7):627630.

5. Buchner A, Shnaiderman A, Vared M. Pediatric localized reactive gingival lesions: a retrospective study from Israel. Pediatr Dent 2010;32(7):486-492.
6. Mishra A, Pandey RK. Fibro-epithelial polyps in children: a report of two cases with a literature review. Intractable Rare Dis Res 2016;5(2):129-132. DOI: 10.5582/irdr.2016.01015.

7. Romero U, Russo C, Palaia G, et al. Biopsy of different oral soft tissues lesions by KTP and diode laser: histological evaluation. ScientificWorldJournal 2014;2014:761704.

8. Bakhtiari S, Taheri JB, Sehhatpour M, et al. Removal of an extra-large irritation fibroma with a combination of diode laser and scalpel. J Lasers Med Sci 2015;6(4):182-184. DOI: 10.15171/jlms.2015.16.

9. Strauss RA. Lasers in oral and maxillofacial surgery. Dent Clin North Am 2000;44(4):851-873.

10. AAPD. Policy on use of lasers for pediatric dental patients. AAPD Reference Manual 2013;36:75-77.

11. Azma E, Safavi N. Diode laser application in soft tissue oral surgery. J Lasers Med Sci 2013;4(4):206-211. 